\title{
Research article (Award paper) \\ Diagnostic accuracy of maternal serum endothelial-derived nitric oxide synthase (eNOS) and its correlation with birth outcomes, in preeclampsia - a case-control study
}

\author{
Sangappa Virupaxappa Kashinakunti ${ }^{1,2}$, Basavaraj Devaranavadagi ${ }^{3}$, Manjula Rangappa ${ }^{4}$, Ashalata Mallapur ${ }^{5}$ \\ ${ }^{1} \mathrm{PhD}$ Scholar, ${ }^{3}$ Professor and Head, Department of Biochemistry, Shri B M Patil Medical College, BLDE University, \\ Vijayapur-586101, Karnataka, India \\ ${ }^{2}$ Professor, Department of Biochemistry, ${ }^{4}$ Professor, Department of Community Medicine, ${ }^{5}$ Professor and Head Department of \\ Obstetrics and Gynaecology, S. Nijalingappa Medical College, Navanagar, Bagalkot-587102 Karnataka, India
}

Author for correspondence: Sangappa V. Kashinakunti. Email: drsvkashinakunti@yahoo.co.in

\begin{abstract}
Introduction and Aim: Preeclampsia (PE) results from impaired placentation, leading to placental hypoxia and dysfunction and abnormalities in the endothelial nitric oxide synthase (eNOS)-nitric oxide pathway. The present study was undertaken to estimate and compare maternal serum eNOS concentrations in women with PE and normal healthy pregnant women, to know the correlation of eNOS level with severity of PE, to find the best cut-off value for the diagnosis of PE and to study its association of eNOS levels with the birth outcome.

Material and Methods: This was a case-control study, conducted at a tertiary care hospital. The study was approved by the Institutional ethics committee. Informed consent was obtained from all the participants. Primigravida (singleton) pregnant women aged 18-35 years, 20 or more weeks of gestation, diagnosed as PE and classified as mild/severe PE based on America college of obstetricians and gynecologists guidelines were selected for the study. eNOS was estimated by the ELISA method. All the participants were visited again to note the outcomes.
\end{abstract}

Results: The maternal serum eNOS in PE was significantly lower than the control group ( $\mathrm{p}=0.019)$. The best cutoff value was $187.25 \mathrm{pg} / \mathrm{ml}$ to diagnose PE, area under the ROC curve (AUC) 0.61 , sensitivity $95.1 \%$, specificity $37.5 \%$ and diagnostic accuracy was $66,67 \%$. Birth outcomes did not have a significant correlation with eNOS.

Conclusion: eNOS was significantly lowerer in PE, it can be used as a diagnostic marker with the best cut of value of $187.25 \mathrm{pg} / \mathrm{ml}$.

Keywords: Preeclampsia; nitric oxide synthase; diagnosis.

\section{INTRODUCTION}

$\mathrm{P}$ reeclampsia (PE), constitute a unique, complex clinical condition of human pregnancy, is characterized by the development of de-novo hypertension and proteinuria after 20 weeks or above gestation in patients free from any clinical disease, but it can occur at any time during labor, or even up to 6 weeks after delivery. $\mathrm{PE}$ is a multi-organ involvement targets the endothelium of the brain, liver, kidneys and the coagulation system and impairs a short-term perinatal outcome and remote prognosis of cardiovascular disease for the mother. Termination of pregnancy remains the only curative treatment in severe PE. Management of patients with PE must be individualized and must balance the maternal and fetal risks (1-3).

It has been suggested that the root cause of PE is the placenta. Its functions are vascular development and blood flow, which depend on proper trophoblast growth and differentiation. According to the studies, preeclampsia results from impaired placentation early in the beginning of the pregnancy, leading to placental hypoxia and dysfunction and abnormalities in the endothelial nitric oxide synthase (eNOS)-nitric oxide pathway (4-6). eNOS is localized mainly in the plasma membrane, and a small amount is present in the cytosol also. Golgi apparatus acts as the main source of eNOS. The eNOS is a key enzyme of the cardiovascular system that contributes to vascular homeostasis through tightly regulated $\mathrm{NO}$ production $(7,8)$. Endothelial cells release NO, a potent vasodilator. Nitric oxide is required for regulation of the vascular tone and hence maintains blood pressure. The placental blood flow is regulated by NO and it also participates in trophoblast invasion and development of the placenta. As NO is a highly reactive and short-lived molecule. It is clearly not known whether eNOS deficiency plays an important role in pathogenesis of preeclampsia. Many studies suggest that insufficient nitric oxide synthesis or NO bioavailability, may contribute to increased blood pressure, systemic vascular resistance, and sensitivity to the pressors. However, studies conducted among various ethnic groups, which resulted in mixed or inconclusive results (9-12).

The current study was undertaken to estimate and compare maternal serum concentrations of endothelial nitric oxide synthase (eNOS) in women with $\mathrm{PE}$ in comparison to healthy normotensive pregnant women. and also to find the correlation between eNOS level with severity of PE, the best 
cutoff value for the diagnosis of PE and to study its association of eNOS levels with the birth outcome.

\section{MATERIALS AND METHODS}

This is a case-control study, conducted in the department of obstetrics and gynecology and department of biochemistry at tertiary care hospital, Karnataka India, from Jan 2019 to Jun 2019. The study was approved by the Institutional ethics committee. Informed consent was obtained from all the study participants.

Primigravida (singleton) pregnant women aged 18-35 years, 20 or more weeks of gestation, diagnosed as $\mathrm{PE}$ and classified as mild/severe PE based on America college of obstetricians and gynecologists' guidelines (13) were selected for the study. Patients with premature rupture of membranes, chorioamnionitis, multiple gestations, $\mathrm{Rh}$ isoimmunization, fetal anomalies, intrauterine fetal death, chronic inflammatory diseases, history of diabetes mellitus, history of systemic hypertension, cardiovascular or renal diseases and chronic inflammatory diseases were excluded from the study. Healthy pregnant women, matched for age, gravida and gestational weeks with the cases were selected as controls.

All participants were subjected to history taking and followed by general physical examination. Abdominal examination was performed for fundal level, lie and presentation of the fetus. Blood samples were collected by venipuncture in two tubes, $2 \mathrm{ml}$ with anticoagulant and $2 \mathrm{ml}$ without anticoagulant. Complete blood count, blood grouping and rhesus factor were done. After clotting, the samples were centrifuged and serum was pipetted and the following parameters were measured blood glucose, liver function tests, urea. serum creatinine, uric acid and eNOS. Sample for eNOs measurement was stored at $20^{\circ} \mathrm{C}$ till estimation. eNOS was estimated by the ELISA method the kits were supplied by Bioassay Technologies Laboratory. Urine analysis for proteinuria was done. All the participants were visited again at the outcome of the pregnancy and the outcome findings were noted.

Sample size calculation was done using Open Epi software version $2.3: 1$, retrospectively with $83.47 \%$ power of the study, using case severity into consideration, the sample size calculated was 39 45 . Hence, 45 preeclampsia cases and 45 normal gestation controls were included in the study.

Analysis was done using SPSS software version 19. Unpaired ' $t$ ' test for quantitative data and Pearson's correlation tests were applied. $\mathrm{P}<0.05$ will be considered statistically significant. ROC curve analysis for eNOS was done to find the optimum cutoff value for the diagnosis of PE. Tests of validity namely sensitivity, specificity, positive predictive value, negative predictive value and diagnostic accuracy of eNOS were calculated with 95\% confidence intervals.

\section{RESULTS}

In the present study, there was no significant difference in maternal age, gestational weeks. The systolic, diastolic blood pressure, SGOT were significantly higher in $\mathrm{PE}$ patients than normal pregnant women (Table 1).

Table 1: Demographic characteristics of controls and cases

\begin{tabular}{|c|c|c|c|}
\hline & Controls & Cases & p \\
\hline Age in years & $22.55 \pm 3.22$ & $22.83 \pm 4.23$ & 0.94 \\
\hline Gestational Weeks & $37.88 \pm 2.87$ & $35.83 \pm 4.77$ & 0.085 \\
\hline SBP mmHg & $118.51 \pm 10.65$ & $158.45 \pm 18.85$ & $\mathbf{0 . 0 0 0}$ \\
\hline DBP mmHg & $75.40 \pm 8.75$ & $102.30 \pm 9.20$ & $\mathbf{0 . 0 0 0}$ \\
\hline Platelet & $228792.84 \pm 49502.22$ & $200888.45 \pm 82528.64$ & 0.078 \\
\hline Creatinine mg/dl & $0.72 \pm 0.14$ & $0.76 \pm 0.10$ & 0.600 \\
\hline AST IU/L & $24.27 \pm 10.38$ & $44.53 \pm 62.24$ & $\mathbf{0 . 0 4 4}$ \\
\hline ALT IU/L & $15.85 \pm 10.18$ & $36.37 \pm 68.25$ & 0.067 \\
\hline Birth weight in Kg & $2.88 \pm 0.67$ & $2.16 \pm 0.85$ & $\mathbf{0 . 0 0 0}$ \\
\hline APGAR 1min & $7.82 \pm 0.770$ & $6.49 \pm 2.62$ & $\mathbf{0 . 0 1 6}$ \\
\hline APGAR 5min & $8.94 \pm 0.40$ & $7.69 \pm 2.85$ & $\mathbf{0 . 0 2 0}$ \\
\hline
\end{tabular}

SBP: Systolic blood pressure, DBP: Diastolic blood pressure, AST: Aspartate transaminase, ALT: Alanine transaminase

Table 2: eNOS in controls and cases

\begin{tabular}{|c|c|c|c|}
\hline & Controls & Cases & p \\
\hline eNOS pg/ml & $173.36 \pm 187.68$ & $99.87 \pm 56.58$ & 0.019 \\
\hline
\end{tabular}

eNOS: Endothelial derived nitric oxide synthase

The maternal serum eNOS in PE was significantly lower than the control group $(\mathrm{p}=0.019)$. 


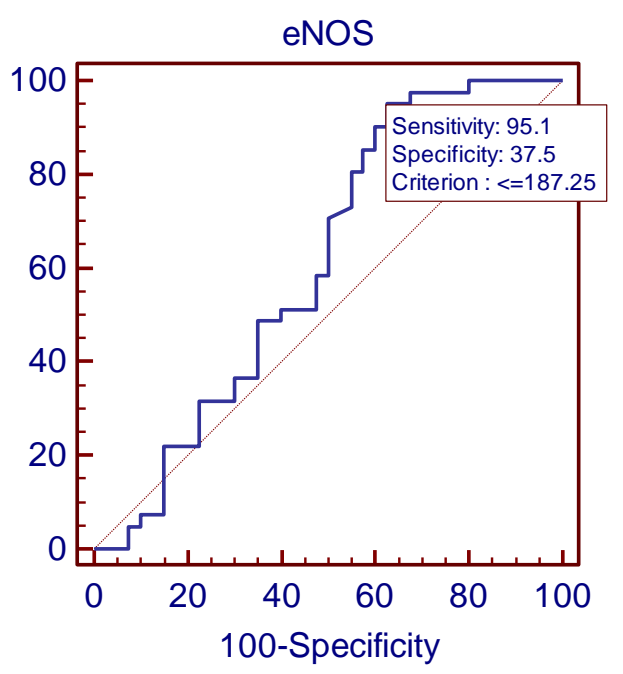

Fig. 1: The best cutoff value of eNOS for the diagnosis of PE by ROC curve

Table 3: Sensitivity, Specificity, Positive Predictive Value, Negative Predictive Value and Diagnostic Accuracy of eNOS in PE patients

\begin{tabular}{|c|c|c|}
\hline Sensitivity & $95.1 \%$ & $\left(48.69,71.94^{1}\right)$ \\
\hline Specificity & $37.5 \%$ & $\left(65.66,96.71^{1}\right)$ \\
\hline Positive Predictive Value & $95.12 \%$ & $\left(83.86,98.65^{1}\right)$ \\
\hline Negative Predictive Value & $37.50 \%$ & $\left(24.22,52.97^{1}\right)$ \\
\hline Diagnostic Accuracy & $66.67 \%$ & $\left(55.85,75.97^{1}\right)$ \\
\hline
\end{tabular}

The best cut-off value was $187.25 \mathrm{pg} / \mathrm{ml}$ to diagnose PE, area under the ROC curve (AUC) 0.61 , sensitivity $95.1 \%$, specificity $37.5 \%$ and diagnostic accuracy was $66.67 \%$ (Fig. 1, Table 3).

Table 4: eNOS in mild and severe PE patients

\begin{tabular}{|c|c|c|c|}
\hline & Mild PE & Severe PE & p \\
\hline eNOS pg/ml & $101.18 \pm 60.63$ & $95.76 \pm 40.94$ & 0.824 \\
\hline
\end{tabular}

eNOS: Endothelial derived nitric oxide synthase, PE: Preeclampsia.

There was decrease in eNOS in severe PE cases than mild PE cases but it was not statistically significant $(\mathrm{p}=0.824)$ (Table 4).

Table 5: Correlation of eNOS with the severity of PE and birth outcomes

\begin{tabular}{|c|c|c|c|c|}
\hline & Severity of PE & Birth weight & APGAR 1min & APGAR 5min \\
\hline $\mathbf{r}$ & 0.036 & -0.029 & -0.112 & -0.142 \\
\hline $\mathbf{p}$ & 0.824 & 0.868 & 0.530 & 0.422 \\
\hline
\end{tabular}

eNOS: Endothelial derived nitric oxide synthase, PE: Preeclampsia

Birth outcomes did not have a significant correlation with eNOS (Table 5).

\section{DISCUSSION}

NO is synthesized from the reduction of L-arginine to L-citrulline by the NOS enzyme, which has three isoforms: nNOS or neuronal, iNOS, the inducible and eNOS endothelial NOS, here BH4 acts as a coenzyme, promotes dimerization and activity of NOS. Decreased concentration of the substrate Larginine and increased concentration of the endogenous NOS inhibitor asymmetric dimethylarginine (ADMA) may interfere with eNOS activity in preeclampsia $(14,15)$.

In the present study, there was a significant decrease in eNOS in PE patients compared to the normal pregnant women $(\mathrm{p}=0.019)$. Kim et al., and Khalil et $a l$., found lower expression of eNOS in the syncytiotrophoblast, reduced concentrations of Larginine, and unchanged ADMA in the serum of women with pregnancies complicated by preeclampsia $(16,17)$. A cohort study conducted in Greek showed that the plasma ADMA levels in PE women were found to be higher (18) than the control group. Maranzana et al., and Laskowska et al., showed that the levels of serum eNOS were lower in women with PE than in the healthy women from the control group, but it was not statistically significant

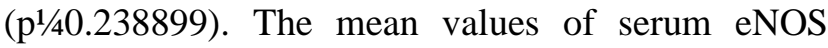
were $181.30 \pm 178.44 \mathrm{U} / \mathrm{ml}$ in the PE group and $217.74 \pm 265,11 \mathrm{U} / \mathrm{ml}$ in the control group $(19,20)$. The levels of serum endothelial nitric oxide synthase were lower in women with pregnancies complicated by severe PE than in the healthy women from the control group, but these differences were not statistically significant $(p=0.118770)$. The mean values of serum eNOS were $134.06 \pm 76.73 \mathrm{U} / \mathrm{ml}$ in the Pre group and $187.70 \pm 165.41 \mathrm{U} / \mathrm{ml}$ in the Control group (21). Myatt et al., (5) observed intense expression of eNOS in placentas from pregnancies 
complicated by preeclampsia. Schiessl et al., found significantly increased placental expression of endothelial nitric oxide synthase in pregnancies complicated by preeclampsia (22). In a Brazilian cohort study, plasma ADMA concentration was more in PE women and plasma NO concentration was found lower in comparison with normotensive women (23).

The uncoupling of eNOS has also been shown as a source of superoxide formation and this leads to reduce NO production. When eNOS cofactor, tetrahydrobiopterin(BH4) is low or when posttranslational changes regulate eNOS function. It has been demonstrated that various inflammatory makers like TNF- $\alpha$ and CRP are increased in plasma and placenta from PE women. TNF- $\alpha$ an inflammatory marker increased in PE, which downregulates eNOS and mitochondrial biogenesis leading to mitochondrial dysfunction and elevated ROS. Conversely, CRP is another inflammatory marker also increased in $\mathrm{PE}$, indirectly downregulates $\mathrm{BH} 4$ production, leading to uncoupling of eNOS catalysed reaction and leads the formation of peroxynitrite. Animal studies have shown that $\mathrm{BH} 4$ supplementation in $\mathrm{pE}$ increased the concentration of NO $(14,15,24)$.

In the present also there was low levels of maternal serum concentration of eNOS in severe PE as compared to mild PE but it was not statistically significant $(\mathrm{p}=0,824)$. The unchanged levels of eNOS in severe PE. A small increase in eNOS in mild hypertension PE patients, whereas in a subgroup with severe hypertension, concentrations of these markers were greatly reduced. Studies have shown elevated ADMA levels in early-onset PE may suggest a relationship between the severity of the disease and determining the time of PE clinical manifestations $(2,12,25)$.

In the current study the best cut-off value was 187.25 $\mathrm{pg} / \mathrm{ml}$ to diagnose PE, area under the ROC curve (AUC) 0.61 , sensitivity $95.1 \%$, specificity $37.5 \%$ and diagnostic accuracy was $66.67 \%$ (Figure 1, Table 3). Wender-Ozegowska et al., in their study, found that eNOS AUC was 0.57 best cut-off was 0.34 with a sensitivity of $78.6 \%$ specificity $40 \%$ (1). Our study did not show any correlation of eNOS with the severity of PE and birth outcome viz birth weight and APGAR score.

Further studies are required with gene expression along with serum estimation of NO, eNOS, Larginine, ASDA, BH4, oxidative stress markers and inflammatory markers may give the role of $\mathrm{NO}$ and eNOS in the pathogenesis of PE.

\section{CONCLUSION}

The eNOS was significantly lower in PE patients than normal healthy pregnant women, it can be used as tool diagnose PE with the best cut-off value of
$187.25 \mathrm{pg} / \mathrm{ml}$, with sensitivity $95.1 \%$, specificity $37.5 \%$ and diagnostic accuracy of $66.67 \%$.

\section{CONFLICT OF INTEREST}

The authors declare no conflict of interest.

\section{REFERENCES}

1. Wender-Ozegowska, E., Zawiejska, A., Lciek, R., Brązert, J. Concentrations of eNOS, VEGF, ACE PlGF in maternal blood as predictors of impaired fetal growth in pregnancy complicated by gestational hypertension/preeclampsia. Hypertension in Pregnancy 2015;34(1):17-23.

2. Laskowska, M., Laskowska, K., Terbosh, M., Oleszczuk, J. A comparison of maternal serum levels of endothelial nitric oxide synthase, asymmetric dimethylarginine, and homocysteine in normal and preeclamptic pregnancies. Med Sci Monit 2013;19: 430-437.

3. Uzan, J., Carbonnel, M., Piconne, O., Asmar, R., Ayoubi, J. M. Pre-eclampsia: pathophysiology, diagnosis, and management. Vasc Health Risk Manag 2011;7: 467-474.

4. Roberts, J.M., Escudero, C. The placenta in Preeclampsia. Pregnancy Hypertens 2012;2(2):72-83.

5. Myatt, L. Review: Reactive oxygen and nitrogen species and functional adaptation of the placenta. Placenta 2010;31(Suppl.): S66-S69.

6. Mislanova, C., Martsenyuk, O., Huppertz, B., Obolenskaya, M. Placental markers of folate-related metabolism in preeclampsia. Reproduction, 2011;142(3):467-476.

7. Sessa WC. eNOS at a glance. J Cell Sci 2004;117: $2427-$ 2429.

8. Shaul, P.W. Regulation of endothelial nitric oxide synthase: location, location, location. Annu Rev Physiol 2002; 64: 749-774.

9. Demir, B., Demir, S., Pasa, S., Guven, S., Atamer, Y., Atamer, A., et al., The role of homocysteine, asymmetric dimethylarginine and nitric oxide in pre-eclampsia. J Obstet Gynaecol 2012;32(6):525-528.

10. Huang, L.T., Hsieh, C.S., Chang, K.A., Tain, Y.L. Roles of nitric oxide and asymmetric dimethylarginine in pregnancy and fetal programming. Int J Mol Sci 2012;13(11):1460614622.

11. Kulandavelu, S., Whiteley, K.J., Qu, D., Mu, J., Bainbridge, S.A., Adamson S.L. Endothelial nitric oxide synthase deficiency reduces uterine blood flow, spiral artery elongation, and placental oxygenation in pregnant mice. Hypertension 2012;60(1):231-238.

12. Zawiejska, A., Wender-Ozegowska, E., Iciek, R., Brazert, J. Concentrations of endothelial nitric oxide synthase, angiotensin-converting enzyme, vascular endothelial growth factor and placental growth factor in maternal blood and maternal metabolic status in pregnancy complicated by hypertensive disorders. Journal of Human Hypertension 2014;28:670-676.

13. American College of Obstetricians and Gynecologists; Task Force on Hypertension in Pregnancy. Hypertension in pregnancy. Report of the American College of Obstetricians and Gynecologists' Task Force on Hypertension in Pregnancy. Obstet Gynecol 2013;122: 1122-1131.

14. Sánchez-Aranguren, L.C., Prada, C.E., Riaño-Medina, C.E., Lopez, M., Endothelial dysfunction and preeclampsia: role of oxidative stress. Frontiers in Physiology 2014 Oct;5(Article372):1-11.

15. Qian, J., Fulton, D. Post-translational regulation of endothelial nitric oxide synthase in vascular endothelium. Front Physiol 2013; 4:347. doi:10. 3389/fphys.2013.00347.

16. Kima, Y.J., Parkc, H.S., Leed, H.Y., Hac, E.H., Suhe, S.H., Ohf, S.K., et al., Reduced L-arginine Level and Decreased Placental eNOS Activity in Preeclampsia. Placenta 2006);27:438-444.

17. Khalil, A.A., Tsikas, D., Akolekar, R., Jordan, J., Nicolaides, K.H. Asymmetric dimethylarginine, arginine 
and homoarginine at 11-13weeks' gestation and preeclampsia: a case-control study. J Hum Hypertens 2013;27:38-43.

18. Savvidis, A., Daniilidis, A., Giannoulis, C., Tantanasis, T., Koiou, K., Zournatzi, V., et al., Correlation between asymmetric dimethylarginine maternal plasma levels and preeclampsia. Clin Exp Obstet Gynecol 2011; 38:134-136.

19. Maranzana, E., Barbero, G., Falasca, A.I., Lenaz, G., Genova, M.L. Mitochondrial respiratory super complex association limits production of reactive oxygen species from complex I. Antioxid Redox Signal 2013;19:1469-1480.

20. Laskowska, M., Laskowska, K., Oleszczuk, J. The relation of maternal serum eNOS, NOSTRIN and ADMA levels with aetiopathogenesis of preeclampsia and/or intrauterine fetal growth restriction. J Matern Fetal Neonatal Med 2014; DOI:10.3109/14767058.2014.900036.

21. Laskowska, M., K. Laskowska, K., Oleszczuk, J. Pregnancy Hypertension: An International Journal of Women's Cardiovascular Health 2012;2:240-339.

22. Schiessl, B., Mylonas, I., Hantschmann, P., Kuhn, C., Schulze, S., Kunze, S., et al., Expression of endothelial NO synthase, inducible NO synthase, and estrogen receptors alpha and beta in placental tissue of normal, preeclamptic, and intrauterine growth-restricted pregnancies. J Histochem Cytochem, 2005; 53(12): 1441-1449.

23. Sandrim, V.C., Palei, A.C., Metzger, I.F., Cavalli, R.C., Duarte, G., Tanus Santos, J.E. Interethnic differences in ADMA concentrations and negative association with nitric oxide formation in preeclampsia. Clin Chim Acta 2010; 411:1457-1460.

24. Yzydorczyk, C., Comte, B., Huyard, F., Cloutier, A., Germain, N., Bertagnolli, M., et al., Developmental programming of eNOS uncoupling and enhanced vascular oxidative stress in adult rats after transient neonatal oxygen exposure. J Cardiovasc. Pharmacol 2013; 61: 8-16.

25. Dymara-Konopka,W., Laskowska, M. The Role of Nitric oxide, ADMA, and homocysteine in the etiopathogenesis of preeclampsia-Review. Int J Mol Sci 2019; 20:2757. 